\title{
Adipositas-Chirurgie entlastet auch Wirbelsäule und Gelenke
}

\author{
Unter der Last einer Adipositas leiden nicht nur der Stoffwechsel und \\ innere Organe, sondern auch die Wirbelsäule sowie die Hüft-, Knie- \\ und Fußgelenke. Eine US-amerikanische Studie bestätigt nun: Die \\ Adipositas-Chirurgie erzielt nicht nur positive Effekte auf den Stoff- \\ wechsel, die Patienten werden auch wieder beweglicher, ausdauern- \\ der und haben weniger Schmerzen.
}

n einer Beobachtungs-Kohortenstudie (Longitudinal Assessment of Bariatric Surgery-2) an zehn US-Kliniken haben Wendy King von der Universität Pittsburgh und ihr Team untersucht, wie sich Schmerzen und körperliche Funktionsfähigkeit in den ersten drei Jahren nach Einsetzen eines Roux-Y-Magenbypass (RYGB) oder eines laparoskopisch verstellbaren Magenbandes (LAGB) verändern. Zur Beurteilung der Erfolge verwendeten die Autoren den 36-ItemShort-Form Health Survey (SF-36). Je höher der Score, desto geringer der Schmerz und desto besser die Funktion. Eine relevante Verbesserung war als Steigerung um mindestens fünf Punkte auf einer Skala von 1-100 definiert. Zudem wurde die Zeit gemessen, die Probanden prä- und postoperativ für eine Gehstrecke von 400 Metern benötigten. Als Erfolg galt hier eine Zeitersparnis von mindestens 24 Sekunden. Sekundäre Endpunkte waren die Verbesserung im Western Ontario McMaster (WOMAC) Osteoarthritis Index um mindestens 9,7 Schmerz- und 9,3 Funktionspunkte auf einer Skala von 0-100.

$70,4 \%$ der Patienten erhielten einen RYGB, 25,0\% ein LAGB. Am Studienende konnten die Daten von 2.221 der insgesamt 2.458 Teilnehmer im Durchschnittsalter von 47 Jahren und mit einem mittleren präoperativen BodyMass-Index (BMI) von $45,9 \mathrm{~kg} / \mathrm{m}^{2}$ ausgewertet werden.

Die größten Vorteile haben Patienten mit besonders schwerer Symptomatik: Bereits ein Jahr nach dem Eingriff hatte bei 57,6 \% der Patienten der körperliche Schmerz nachgelassen. Der SF-36-Score war signifikant von anfänglich durchschnittlich 39,9 auf 47,3 Punkte gestiegen. Bei $76,5 \%$ hatte sich die körperliche Funktionsfähigkeit verbessert (im SF-
36 -Score von 36,6 auf 49,2), und 59,5\% konnten die 400-m-Strecke schneller absolvieren als vor der Operation.

Zum Ende des dritten Jahres sanken die Erfolge wieder etwas ab: Der Körperschmerz war dann bei 48,6 \% der Patienten geringer (SF-36: 44,8), die körperliche Funktion bei $70,2 \%$ besser (SF-36: 47,8) als zu Studienbeginn. Doch trotz dieser Einbußen blieben die erzielten Erfolge auch nach dem dritten Jahr signifikant über den Ausgangswerten. Erhalten blieben nach drei Jahren die Verbesserungen der Gehzeit, der Knie- und Hüftschmerzen sowie der Funktionsfähigkeit dieser Gelenke. Im WOMACScore sanken die Mittelwerte für Knieschmerzen bis zum dritten Jahr von 46,5 auf 26,2 und für Hüftschmerzen von 47,4 auf 25,7. Ähnliche Verbesserungen wurden bezüglich der Funktion dieser Gelenke erzielt werden (Knie von 48,6 auf 24,6; Hüfte von 46,7 auf 22,2).

Besonders profitierten jene Adipösen, die zu Studienbeginn und damit präoperativ unter starken Schmerzen und Unbeweglichkeit in Knie- und Hüftgelenken gelitten hatten. Bei etwa drei Viertel dieser Patienten mit Arthrosesymptomatik hatten sich nach einem Jahr der Knieschmerz $(77,1 \%)$ und die -beweglichkeit $(78,6 \%)$ sowie der Hüftschmerz $(74,1 \%)$ und die -funktion $(79,2 \%)$ verbessert.

Mit dem Nachlassen der Schmerzen sank auch der Verbrauch an Schmerzmitteln signifikant. Allerdings war der Analgetikakonsum wegen Rücken- oder Beinschmerzen in dieser Patientengruppe auch drei Jahre nach der Operation immer noch höher als in der Allgemeinbevölkerung.
Fazit: Die deutliche Gewichtsreduktion nach Einsetzen eines Roux-Y-Magenbypass (RYGB) oder eines laparoskopisch verstellbaren Magenbandes (LAGB) bei Patienten mit exzessiver Adipositas wirkte sich auch günstig auf die präoperativ vorhandenen Rücken- und Gelenkbeschwerden der unteren Extremitäten aus. Längere Studien sind den Autoren zufolge erforderlich, um die Nachhaltigkeit der positiven Effekte auf die Schmerzen und die Gelenkfunktion zu prüfen.

Christine Starostzik

Wendy C. King et al. Change in Pain and Physical Function Following Bariatric Surgery for Severe Obesity. JAMA 2016; 315(13): 1362-71; doi:10.1001/jama.2016.3010 